\title{
Propagação vegetativa de Hyptis leucocephala Mart. ex Benth. e Hyptis platanifolia Mart. ex Benth. (Lamiaceae)
}

OLIVEIRA, L.M. ${ }^{1 *}$; NEPOMUCENO, C.F. ${ }^{1}$ FREITAS, N.P. ${ }^{1}$; PEREIRA, D.M.S. ${ }^{1}$; SILVA, G.C. ${ }^{1}$; LUCCHESE, A.M. ${ }^{2}$ ${ }^{1}$ Unidade Experimental Horto Florestal, Departamento de Ciências Biológicas, Universidade Estadual de Feira de Santana (UEFS), Avenida Presidente Dutra, Santa Mônica, CEP: 44.077-760, Feira de Santana-Brasil *lenaldo@uefs.br 2'Universidade Estadual de Feira de Santana (UEFS), Departamento de Exatas, Avenida Transnordestina, S/N, Novo Horizonte, CEP: 44.036.900, Feira de Santana-Brasil

RESUMO: O gênero Hyptis (Lamiaceae) apresenta metabolismo especial de notável variabilidade, possuindo predominância de óleos essenciais, que têm muito valor junto a diversas comunidades que utilizam essas plantas pelas propriedades terapêuticas. Diversos estudos com esse gênero têm demonstrado que_os óleos essenciais apresentam propriedades antimicrobiana, antifúngica, citotóxica, antiinflamatória, anti-HIV e inseticida, entretanto, poucos são os estudos voltados para a exploração sustentável dessas espécies. O objetivo desse trabalho foi avaliar o efeito de diferentes tipos de estaca e diferentes concentrações de ácido indolbutírico (AIB) sobre a taxa de sobrevivência, crescimento e enraizamento de estacas de Hyptis leucocephala Mart. ex Benth. e Hyptis platanifolia Mart. ex Benth., espécies endêmicas do semi-árido nordestino cujo valor medicinal tem sido amplamente comprovado. O experimento teve duração de quatro meses. Foram utilizadas estacas apicais, médio-apicais, médio-basais e basais tratadas com soluções de 0,1000, 2000 e $4000 \mathrm{mg} \mathrm{L}^{-1}$ de ácido indolbutírico (AIB), durante 5 minutos. A utilização de estacas apicais, médio/apicais e médio/basais induzidas com 2000 e $4000 \mathrm{mg} \mathrm{L}^{-1}$ de AIB possibilitaram melhores resultados para propagação vegetativa de Hyptis leucocephala. Para Hyptis platanifolia os melhores resultados foram obtidos com a utilização de estacas médio/ basais e basais tratadas com AIB na concentração de 2000 e $4000 \mathrm{mg} \mathrm{L}^{-1}$.

Palavras-chave: Estaquia, produção de mudas, plantas aromáticas, óleos essenciais

\begin{abstract}
Vegetative propagation of Hyptis leucocephala Mart. ex Benth. and Hyptis platanifolia Mart. ex Benth. The genus Hyptis (Lamiaceae) has a special metabolism with remarkable variability and predominance of essential oils of great value to the various communities that use these plants due to their therapeutic properties. A number of studies on this genus have demonstrated that its essential oils present antimicrobial, antifungal, cytotoxic, anti-inflammatory, anti-HIV and insecticidal activities. However, few studies have focused on the sustainable exploitation of these species. The aim of this study was to evaluate the effect of different types of cuttings and different concentrations of indolebutyric acid (IBA) on the survival, growth and rooting rates of cuttings from Hyptis leucocephala Mart. ex Benth. and Hyptis platanifolia Mart. ex Benth., species endemic to the semi-arid region of Northeastern Brazil and with largely proven medicinal value. The experiment lasted four months. Apical, middle-apical, middle-basal and basal cuttings were treated with 0, 1000, 2000 and $4000 \mathrm{mg} \mathrm{L}^{-1}$ IBA solutions for $5 \mathrm{~min}$. The use of apical, middleapical and middle-basal cuttings induced by 2000 and $4000 \mathrm{mg} \mathrm{L}^{-1}$ IBA led to the best results for vegetative propagation of $H$. leucocephala. For $H$. platanifolia, the best results were obtained using middle-basal and basal cuttings treated with IBA at 2000 and $4000 \mathrm{mg} \mathrm{L}^{-1}$.
\end{abstract}

Key words: Cutting, seedling production, aromatic plants, essential oils

\section{INTRODUÇÃO}

A família Lamiaceae é composta por cerca de 250 gêneros e 6.970 espécies (Judd et al., 1999). Muitas espécies dessa família são endêmicas do semi-árido nordestino, com destaque para o gênero Hyptis. O metabolismo especial das plantas do gênero Hyptis é de notável variabilidade, apresentando

Recebido para publicação em 05/07/2009

Aceito para publicação em 05/07/2010

Rev. Bras. PI. Med., Botucatu, v.13, n.1, p.73-78, 2011. 
predominância de óleos essenciais, que têm muito valor junto a diversas comunidades que as usam pelas propriedades terapêuticas. Falcão \& Menezes (2003) citam 25 espécies desse gênero estudadas sob o aspecto farmacológico, descrevendo a presença de substâncias com atividades antimicrobiana, antifúngica, citotóxica, antiinflamatória, anti-HIV e inseticida.

Hyptis leucocephala Mart. ex Benth. é uma erva aromática decumbente de cerca de $20 \mathrm{~cm}$ de altura, apresenta folhas cartáceas, flores com cálice esverdeado e corola arroxeada, ocorrente no semiárido baiano. Estudos fitoquímicos têm revelado rendimento médio de $0,2 \%$ de óleo nas folhas com formiato de isobornila como composto majoritário e atividade antimicrobiana frente a Bacilus cereus, Staphylococcus aureus e Candida albicans (Lucchese et al., 2005). Hyptis platanifolia Mart. ex Benth. é uma planta perene com caule herbáceo ramoso, folha membranosa peciolada e flores com cálice tubuloso e corola branca, que cresce no nordeste brasileiro. Análises fitoquímicas e fitofarmacológicas têm revelado rendimento médio de $0,5 \%$ de óleo nas folhas, com atividade frente à Bacillus cereus, Staphylococcus aureus, Salmonella choleraesuis e Candida albicans, com $\alpha$-farneseno e $\gamma$-bisaboleno como constituintes majoritários (Lucchese et al., 2005; 2006).

Tendo em vista a distribuição restrita dessas espécies e o elevado potencial fitoquímico e econômico para a população do semi-árido, torna-se importante o desenvolvimento de pesquisas voltadas para a propagação e avaliação do potencial produtivo dessas espécies sob condições de cultivo. Hyptis leucocephala e Hyptis platanifolia vêm sendo alvo de estudos fitoquímicos e agronômicos na Universidade Estadual de Feira de Santana, Bahia, entretanto, ainda não existem trabalhos publicados sobre a propagação dessas espécies. Para Momenté et al. (2003), o estudo da propagação de espécie vegetal é um dos pontos de partida na elaboração de tecnologias agrícolas voltadas para o estabelecimento de sistemas de exploração sustentável. Quando se trata de espécies nativas, com pouco conhecimento agronômico, é necessária a identificação dos fatores que afetam a germinação e/ou propagação vegetativa (Blank et al., 2003).

A propagação natural do gênero Hyptis se dá por sementes, entretanto, a produção comercial de mudas por via sexual é limitada em virtude da dormência das sementes (Maia et al., 2008), além da sazonalidade na produção, com redução rápida da viabilidade. Além disso, quando se objetiva a multiplicação de genótipos selecionados, a propagação por sementes promove grande variação, o que torna relevante o desenvolvimento de metodologias para propagação vegetativa.
A estaquia é um dos principais métodos de propagação vegetativa, sendo amplamente utilizado para espécies frutíferas, medicinais e ornamentais. Consiste na retirada de segmentos caulinares da planta-mãe que, sob condições adequadas, emitem raízes, formando nova planta idêntica àquela que lhe deu origem (Hartmann et al., 2002). Para a maioria das espécies o estímulo à emissão de raízes depende da aplicação exógena de auxinas. A auxina sintética aplicada exogenamente mais utilizada no enraizamento de estacas é o ácido indolbutírico (AIB) (Pasqual, 2001; Oliveira et al., 2008; Figueiredo et al., 2009).

A depender da espécie, o tipo de estaca pode influenciar a taxa de enraizamento e sobrevivência. Para as espécies Passiflora alata Dryand. e Passiflora edulis f flavicarpa O. Deg., estacas medianas e basais apresentam melhores taxas de enraizamento comparadas à apical (Salomão et al., 2002). Em contrapartida, Oliveira et al. (2008) sugerem o uso de estacas apicais na propagação vegetativa de alecrim-pimenta (Lippia sidoides Cham.).

Assim, o presente trabalho teve por objetivo a avaliação do efeito do tipo de estaca e da concentração de ácido indolbutírico (AIB) sobre a taxa de sobrevivência, crescimento e enraizamento de estacas de $H$. leucocephala e $H$. platanifolia.

\section{MATERIAL E MÉTODO}

O trabalho foi realizado durante o período de setembro a dezembro de 2007, na Unidade Experimental Horto Florestal da Universidade Estadual de Feira de Santana, Bahia, Brasil, localizada a $12^{\circ} 16^{\prime} 00^{\prime \prime}$ de latitude sul e 385'00" de longitude oeste, apresentando altitude de 234 metros e temperatura média anual de $24^{\circ} \mathrm{C}$. Exsicatas das espécies Hyptis leucocephala Mart. ex Benth e Hyptis platanifolia Mart. ex Benth foram herborizadas no herbário desta universidade (Vouchers número 131323 e 115466, respectivamente).

O experimento foi conduzido em blocos casualizados, utilizando-se quatro repetições por tratamento, com 20 estacas por parcela, constituindo fatorial simples $4 \times 4$ (concentrações de AIB $x$ tipos de estacas). Foram utilizadas estacas apicais (parte apical do ramo), médio-apicais (quarto médio próximo ao ápice do ramo), médio-basais (quarto médio próximo à base do ramo) e basais (parte basal do ramo) de $H$. leucocephala e $H$. platanifolia (Lamiaceae). As estacas foram colhidas de plantas mantidas na coleção de plantas aromáticas do Horto Florestal da UEFS em fase de florescimento, no mês de setembro de 2007. Todas as estacas foram padronizadas com aproximadamente $12 \mathrm{~cm}$, apresentado três nós e um par de folhas, sendo estas cortadas pela metade, medindo aproximadamente 0,5

Rev. Bras. PI. Med., Botucatu, v.13, n.1, p.73-78, 2011. 
cm para $H$. leucocephala e $2,5 \mathrm{~cm}$ para $H$. platanifolia. Após o preparo, as estacas foram tratadas com solução de ácido indolbutírico (AIB), mergulhando-se a base das estacas em soluções com concentrações de 1000, 2000 e $4000 \mathrm{mg} \mathrm{L}^{-1}$, durante um período de 5 minutos. O tratamento testemunha constituiu-se de imersão da base das estacas em água destilada. Optou-se pela avaliação de maior amplitude de concentração de AIB em virtude de ausência de informações sobre a utilização de auxinas no enraizamento de plantas desse gênero, bem como, em virtude dos resultados obtidos por Pimenta et al. (2007), com seis espécies não domesticadas de Lippia (Verbenaceae), onde verificaram baixa eficiência do AIB sobre o enraizamento de estacas, sugerindo a utilização de outras fontes e concentrações de auxinas ou de cofatores para o enraizamento dessas espécies.

Após a imersão, as estacas foram plantadas em bandejas de polipropileno com 128 células contendo mistura de areia e vermiculita na proporção de 1:1 (v/v) como substrato. O material foi mantido em casa-de-vegetação com luminosidade de $75 \%$ e nebulização intermitente por 60 dias e, ao final, avaliou-se a porcentagem de estacas vivas, o número de folhas por brotação, o comprimento das brotações e a porcentagem de estacas enraizadas. Os resultados foram submetidos à análise de variância, testando-se as médias pelo teste de Scott \& Knott a $5 \%$ de probabilidade, utilizando o software SISVAR (Ferreira, 2004).

\section{RESULTADO E DISCUSSÃO}

Para $H$. leucocephala verificou-se maior percentual de estacas vivas com a ultilização de estacas médio-apicais e médio-basais tratadas com 2000 e $4000 \mathrm{mg} \mathrm{L}^{-1}$ de AIB e estacas apicais com $4000 \mathrm{mg} \mathrm{L}^{-1}$ de AIB; maior número de folhas por brotação com estacas apicais, médio-apicais e médiobasais tratadas com 2000 e $4000 \mathrm{mg} \mathrm{L}^{-1}$ de AIB; maior comprimento das brotações com estacas apicais tratadas com 2000 e $4000 \mathrm{mg} \mathrm{L}^{-1}$ de AIB e maior taxa de enraizamento com estacas médio-apicais e médiobasais tratadas com 2000 e $4000 \mathrm{mg} \mathrm{L}^{-1}$ de AIB e estacas apicais com $4000 \mathrm{mg} \mathrm{L}^{-1}$ de AIB (Tabela 1).

TABELA 1. Efeito do tipo de estaca e da concentração de ácido indolbutírico (AIB) sobre o percentual de estacas vivas, número de folhas por brotação, comprimento da brotação e percentual de estacas enraizadas de Hyptis leucocephala Mart. ex Benth. Feira de Santana, BA, 2009.

\begin{tabular}{ccccc}
\hline & \multicolumn{4}{c}{ Tipo de estaca } \\
\cline { 2 - 5 } & Apical & Médio-apical & Médio-basal & Basal \\
\hline 0 & $25,00 \mathrm{aB}$ & $0,00 \mathrm{bB}$ & $37,50 \mathrm{aB}$ & $0,00 \mathrm{bB}$ \\
1000 & $12,50 \mathrm{bB}$ & $6,25 \mathrm{bB}$ & $50,00 \mathrm{aB}$ & $43,75 \mathrm{aA}$ \\
2000 & $62,50 \mathrm{bA}$ & $81,25 \mathrm{aA}$ & $93,75 \mathrm{aA}$ & $37,50 \mathrm{bA}$ \\
4000 & $81,25 \mathrm{aA}$ & $93,75 \mathrm{aA}$ & $75,00 \mathrm{aA}$ & $37,50 \mathrm{bA}$ \\
\hline \multicolumn{5}{c}{ Número de folhas por brotação* } \\
0 & $6,65 \mathrm{aB}$ & $0,00 \mathrm{aB}$ & $5,45 \mathrm{aA}$ & $0,00 \mathrm{aA}$ \\
1000 & $4,25 \mathrm{bB}$ & $2,25 \mathrm{bB}$ & $10,37 \mathrm{aA}$ & $9,92 \mathrm{aA}$ \\
2000 & $15,47 \mathrm{aA}$ & $12,00 \mathrm{aA}$ & $10,30 \mathrm{aA}$ & $5,12 \mathrm{bA}$ \\
4000 & $14,45 \mathrm{aA}$ & $14,07 \mathrm{aA}$ & $12,12 \mathrm{aA}$ & $5,55 \mathrm{bA}$ \\
\hline \multicolumn{5}{c}{ Comprimento da brotação (cm $)^{*}$} \\
0 & $7,00 \mathrm{aB}$ & $0,00 \mathrm{bB}$ & $4,75 \mathrm{aA}$ & $0,00 \mathrm{bA}$ \\
1000 & $1,87 \mathrm{aB}$ & $1,00 \mathrm{aB}$ & $6,37 \mathrm{aA}$ & $4,50 \mathrm{aA}$ \\
2000 & $16,62 \mathrm{aA}$ & $7,37 \mathrm{bB}$ & $7,87 \mathrm{bA}$ & $5,25 \mathrm{bA}$ \\
4000 & $11,75 \mathrm{aA}$ & $8,00 \mathrm{aA}$ & $6,00 \mathrm{bA}$ & $2,50 \mathrm{bA}$ \\
& & Percentual de estacas enraizadas* & \\
0 & $25,00 \mathrm{aB}$ & $0,00 \mathrm{bB}$ & $37,50 \mathrm{aB}$ & $0,00 \mathrm{bB}$ \\
1000 & $12,50 \mathrm{bB}$ & $6,25 \mathrm{bB}$ & $50,00 \mathrm{aB}$ & $43,75 \mathrm{aA}$ \\
2000 & $37,50 \mathrm{bB}$ & $87,50 \mathrm{aA}$ & $75,00 \mathrm{aA}$ & $31,25 \mathrm{bB}$ \\
4000 & $81,25 \mathrm{aA}$ & $93,75 \mathrm{aA}$ & $75,00 \mathrm{aA}$ & $31,25 \mathrm{bB}$ \\
\hline
\end{tabular}

*Médias seguidas pelas mesmas letras minúsculas nas linhas e maiúsculas nas colunas não diferem estatisticamente a 0,05\% de probabilidade de erro pelo teste de Scott-Knott.

Rev. Bras. Pl. Med., Botucatu, v.13, n.1, p.73-78, 2011. 
Para H. platanifolia obteve-se maior percentual de estacas vivas com a ultilização de estacas basais submetidas à imersão em solução com 2000 e 4000 $\mathrm{mg} \mathrm{L}^{-1}$ de AIB e estacas médio-basais submetidas a $2000 \mathrm{mg} \mathrm{L}^{-1}$ de AIB. Quanto ao número de folhas por brotação, os melhores resultados foram obtidos quando se utilizou estacas médio-basais sem AIB, seguido de estacas médio-apicais, médio-basais e basais sob todas concetrações de AIB testadas. Em relação ao comprimento da brotação, os melhores resultados foram obtidos quando estacas médio-basais foram tratadas com $4000 \mathrm{mg} \mathrm{L}^{-1}$ de AIB e estacas basais foram tratadas com 2000 e $4000 \mathrm{mg} \mathrm{L}^{-1}$ de AlB. A porcentagem de enraizamento foi maior em estacas basais tratadas com 2000 e $4000 \mathrm{mg} \mathrm{L}^{-1} \mathrm{e}$ estacas médio-basais com $2000 \mathrm{mg} \mathrm{L}^{-1}$ de AIB (Tabela 2).

Garbuio et al. (2007) observaram que em patchouli (Pogostemon cablin Blanco ex. Benth.), uma espécie herbácea, o número de raízes emitidas por estaca decresceu das estacas apicais para as medianas e destas para as basais. Bona (2005) e Signor et al. (2007) também verificaram que para as espécies Origanum vulgare L., Baccharis articulata Lam. e Baccharis stenocephala Baker, há maior eficiência no enraizamento com a utilização de estacas apicais, possibilitando maior taxa de sobrevivência. Por outro lado, Frazon et al. (2004) verificaram que estacas retiradas da porção basal de ramos de goiabeira-serrana (Acca sellowiana Berg) proporcionaram melhores resultados de enraizamento e sobrevivência em comparação a estacas retiradas de porções apicais. Resultados semelhantes também foram observados por Nicoloso et al. (1999) trabalhando com fáfia (Pfaffia glomerata (Spreng.) Pedersen) e por Fischer et al. (2008), trabalhando com a espécie lenhosa Vaccinium ashei Reade.

Para Fachinello et al. (2005) a variação na capacidade de enraizamento ao longo do ramo da planta ocorre porque há variação na concentração de fitormônios, influenciando o potencial de enraizamento da estaca. Taiz \& Zeiger (2004) afirmam que posições inferiores do ramo são menos favoráveis à diferenciação radicular, pois a região basal apresenta maior grau de lignificação e menor conteúdo de auxinas, já que o ápice caulinar é um conhecido local de síntese desses hormônios. Entretanto, esses mesmos autores ressaltam que a distribuição de hormônios é muito dependente do hábito de

TABELA 2. Efeito do tipo de estaca e da concentração de ácido indolbutírico (AIB) sobre o percentual de estacas vivas, número de folhas por brotação, comprimento da brotação e percentual de estacas enraizadas de Hyptis platanifolia Mart. ex Benth. Feira de Santana, BA, 2009.

\begin{tabular}{|c|c|c|c|c|}
\hline \multirow{2}{*}{$A \mid B\left(m g ~ L^{-1}\right)$} & \multicolumn{4}{|c|}{ Tipo de estaca } \\
\hline & Apical & Médio-apical & Médio-basal & Basal \\
\hline & \multicolumn{4}{|c|}{ Percentual de estacas vivas ${ }^{\star}$} \\
\hline 0 & $0,00 \mathrm{bA}$ & $31,25 \mathrm{aA}$ & $31,25 \mathrm{aB}$ & $18,75 \mathrm{aB}$ \\
\hline 1000 & $0,00 \mathrm{bA}$ & $31,25 \mathrm{aA}$ & $41,66 \mathrm{aB}$ & $37,50 \mathrm{aB}$ \\
\hline 2000 & $0,00 \mathrm{cA}$ & $50,00 \mathrm{bA}$ & $75,00 \mathrm{aA}$ & $87,50 a A$ \\
\hline \multirow[t]{2}{*}{4000} & $6,25 \mathrm{bA}$ & 31,25 aA & $30,50 \mathrm{aB}$ & $62,50 \mathrm{aA}$ \\
\hline & \multicolumn{4}{|c|}{$\mathrm{N}^{0}$ de folhas por brotação* } \\
\hline 0 & $0,00 \mathrm{bA}$ & $2,37 \mathrm{bA}$ & $6,37 \mathrm{aA}$ & $2,00 \mathrm{bA}$ \\
\hline 1000 & $0,00 \mathrm{bA}$ & $2,92 \mathrm{aA}$ & $2,75 \mathrm{aB}$ & $3,37 \mathrm{aA}$ \\
\hline 2000 & $0,00 \mathrm{bA}$ & 4,35 aA & $2,02 \mathrm{bB}$ & 3,77 aA \\
\hline \multirow[t]{2}{*}{4000} & $0,25 \mathrm{bA}$ & 2,75 aA & $3,37 \mathrm{aB}$ & $3,82 \mathrm{aA}$ \\
\hline & \multicolumn{4}{|c|}{ Comprimento da brotação $(\mathrm{cm})^{*}$} \\
\hline 0 & $0,00 \mathrm{aA}$ & $2,12 \mathrm{aA}$ & $2,12 \mathrm{aB}$ & $0,47 \mathrm{aB}$ \\
\hline 1000 & $0,00 \mathrm{bA}$ & $2,37 \mathrm{aA}$ & $4,37 \mathrm{aB}$ & $2,75 \mathrm{aB}$ \\
\hline 2000 & $0,00 \mathrm{bA}$ & $4,75 \mathrm{aA}$ & $3,87 \mathrm{aB}$ & $5,25 \mathrm{aA}$ \\
\hline \multirow[t]{2}{*}{4000} & $1,50 \mathrm{bA}$ & $1,62 \mathrm{bA}$ & 5,92 aA & $5,37 \mathrm{aA}$ \\
\hline & \multicolumn{4}{|c|}{ Percentual de estacas enraizadas* } \\
\hline 0 & $0,00 \mathrm{bA}$ & $31,25 \mathrm{aA}$ & $25,00 \mathrm{aB}$ & $18,70 \mathrm{aB}$ \\
\hline 1000 & $0,00 \mathrm{bA}$ & $31,25 \mathrm{aA}$ & $33,75 \mathrm{aB}$ & $25,00 \mathrm{aB}$ \\
\hline 2000 & $0,00 \mathrm{bA}$ & $37,50 \mathrm{bA}$ & $68,75 \mathrm{aA}$ & $75,00 \mathrm{aA}$ \\
\hline 4000 & $6,25 \mathrm{bA}$ & $31,25 \mathrm{aA}$ & $37,50 \mathrm{aB}$ & 56,75 aA \\
\hline
\end{tabular}

\footnotetext{
*Médias seguidas pelas mesmas letras minúsculas nas linhas e maiúsculas nas colunas não diferem estatisticamente a $0,05 \%$ de probabilidade de erro pelo teste de Scott-Knott.
}

Rev. Bras. Pl. Med., Botucatu, v.13, n.1, p.73-78, 2011. 
crescimento da planta, herbáceo ou lenhoso, o que pode justificar a diversidade de respostas entre as diferentes espécies. Assim, apesar das espécies $H$. leucocephala e H. platanifolia serem herbáceas e de crescimento prostrado as diferentes respostas podem estar relacionadas ao comprimento das ramificações, significativamente maior em $\mathrm{H}$. platanifolia, chegando a 3,0 metros de comprimento, enquanto que em $\mathrm{H}$. leucocephala raramente atingem 0,5 metros.

Os resultados obtidos para número de folhas por brotação em $H$. platanifolia, maior nas estacas retiradas de regiões mais próximas da base, concorda com os resultados obtidos por Nicoloso et al. (1999) trabalhando com fáfia (Pfaffia glomerata), entretanto, é contrastante em relação aos resultados obtidos com $H$. leucocephala nesse trabalho, onde maior número de folhas foi obtido em estacas retiradas de regiões mais próximas do ápice. Para $H$. platanifolia a posição da estaca teve forte influência na emissão de novas folhas, tanto em estacas tratadas quanto não tratadas com AIB, embora esse efeito tenha sido maior na ausência desse regulador, com maior emissão de folhas nas estacas médio-basais (Tabela 2). Essa resposta deve estar relacionada à distância entre o ápice e a base do ramo, em virtude do significativo comprimento do mesmo. A presença de folhas possibilita a produção de fotoassimilados e fitormônios, essenciais para emissão de raízes e crescimento das brotações, sobretudo para estacas pequenas, com reduzida quantidade de reserva, como ocorre com estacas de plantas medicinais herbáceas, a exemplo das Hyptis.

Verifica-se ainda nos resultados obtidos, relação direta entre o número de folhas produzidas por brotação, o comprimento das brotações e a capacidade de enraizamento das estacas, demonstrando efetivo papel das folhas na geração de substâncias que favorecem a emissão de raízes nas estacas. Esses resultados concordam com Nicoloso et al. (1999), que consideram as folhas produzidas durante o processo de enraizamento como fator chave na emissão de raízes. Fachinello et al. (2005) atribuem à produção de fitormônios, tal como ácido indolácetico, e de cofatores a grande importância das folhas no processo de enraizamento de estacas.

Outro aspecto relevante dos resultados obtidos nesse trabalho foi efeito positivo de concentrações relativamente altas de AIB na sobrevivência e enraizamento de estacas de $H$. leucocephala e H. platanifolia. Ramos et al. (2003) também verificaram que a concentração de $2000 \mathrm{mg}$ $\mathrm{L}^{-1}$ de AIB promoveu os melhores resultados em relação ao número de raízes emitidas por estaca, comprimento médio das brotações e biomassa seca das raízes de mirabolano (Prunus cerasifera Ehrh.). Para esses autores, as auxinas são essenciais no processo de enraizamento, possivelmente por estimularem a síntese de etileno, favorecendo, assim, à emissão de raízes. Por outro lado, Pasqual (2001) afirma que, independente da fórmula estrutural da auxina, a excessiva concentração desse regulador pode ser tóxica à planta, contribuindo também à formação de calos na base das estacas, comprometendo a rizogênese e o crescimento da parte aérea. Contudo, efeitos tóxicos e formação de calos na base das estacas não foram constatados nesse trabalho nas concentrações utilizadas.

Pimenta et al. (2007), trabalhando com estaquia de quatro diferentes quimiotipos de Lippia alba (Mill.) com utilização de diferentes auxinas, verificaram que o AIB foi a auxina mais eficiente na indução de rizogênese nas estacas, em contraste ao ácido indolacético (AIA), que estimulou o enraizamento em menor intensidade. Para Hartman et al. (2002), essa maior capacidade do AIB pode ser atribuída a estrutura química, menos propensa à ação catalítica causada pela ação das enzimas de degradação, naturalmente presentes nas estacas.

Diante dos resultados obtidos nesse trabalho, conclui-se que a estaquia é uma metodologia adequada para propagação vegetativa de $H$. leucocephala e $H$. platanifolia, mediante a utilização de estacas apicais, médio-apicais ou médio-basais tratadas com 2000 ou $4000 \mathrm{mg} \mathrm{L}^{-1}$ AlB para $\mathrm{H}$. leucocephala e estacas médio-basais e basais tratadas com 2000 ou 4000 $\mathrm{mg} \mathrm{L}^{-1}$ AIB para $H$. platanifolia. Entretanto, considerando-se que os valores obtidos nessas concentrações são bastante próximos e buscando-se redução nos custos para produção de mudas recomenda-se a utilização de $2000 \mathrm{mg} \mathrm{L}^{-1}$ AlB para propagação via estaquia de ambas as espécies.

\section{REFERÊNCIA}

BLANK, A.F. et al. Produção de mudas de sambacaitá (Hyptis pectinata L. Poit) em função de recipientes, composição de substratos e calcário. Horticultura Brasileira, v.21, n.1, p.1-4, 2003.

BONA, C.M. et al. Estaquia de três espécies de Baccharis. Ciência Rural, v.35, n.1, p.223-6, 2005.

FACHINELLO, J.C. et al. Propagação vegetativa por estaquia. In: FACHINELLO, J.C. et al. Propagação de plantas frutíferas. Brasília: Embrapa Informações Tecnológicas, 2005. 109p.

FALCÃO, D.Q.; MENEZES, F.S. Revisão etnofarmacológica, farmacológica e química do gênero Hyptis. Revista Brasileira de Farmacognosia, v.84, n.3, p.69-74, 2003.

FERREIRA, D.F. SISVAR - Sistema de análises estatísticas. Versão 4.6. - Lavras: DEX/UFLA, 2004. 32p. FIGUEIREDO, L.S. et al. Influência do ácido indolbutírico no enraizamento de alecrim-pimenta (Lippia sidoides) em leito com umidade controlada. Revista Brasileira de Plantas Medicinais, v.11, n.1, p.33-6, 2009.

FISCHER, D.L.O. et al. Efeito do ácido indolbutírico e da

Rev. Bras. Pl. Med., Botucatu, v.13, n.1, p.73-78, 2011. 
cultivar no enraizamento de estacas lenhosas de mirtilo. Revista Brasileira Fruticultura, v.30, n.2, p.285-9, 2008. FRAZON, R.C.;ANTUNES, L.E.C.; RASEIRA, M.C.B. Efeito do AIB e de diferentes tipos de estaca na propagação vegetativa de goiabeira-serrana (Acca selowiana Berg.). Revista Brasileira de Agrociência, v.10, n.4, p.515-8, 2004. GARBUIO, C. et al. Propagação por estaquia em patchouli com diferentes números de folhas e tipos de estacas. Scientia Agrária, v.8, n.4, p.435-8, 2007.

HARTMANN, H.T. et al. Plant propagation: principles and practices. 7.ed. New Jersey: Prentice Hall International, 2002. 770p.

JUDD, W.S. et al. Plant systematics: a phylogenetic approach. Sunderland: Sinauer Associates, 1999. 463p. LUCCHESE, A.M. et al. Óleos essenciais do gênero Hyptis da região do semi-árido da Bahia. In: SIMPÓSIO BRASILEIRO DE ÓLEOS ESSENCIAS, 3., 2005, Campinas. Anais... Campinas: Instituto Agronômico de Campinas, 2005. p.118.

LUCCHESE, A.M. et al. Comparação da atividade antimicrobiana de óleos essenciais extraídos de espécies do semi-árido baiano. In: REUNIÃO ANUAL DA SOCIEDADE BRASILEIRA DE QUÍMICA, 29., 2006, Águas de Lindóia. Anais... Águas de Lindóia: Sociedade Brasileira de Química, 2006. p.285.

MAIA, S.S.S. et al. Germinação de sementes de Hyptis suaveolens (I.) Poit. (Lamiacea) em função da luz e da temperatura. Caatinga, v.21, n.4, p.212-8, 2008.
MOMENTÉ, V.G. et al. Crescimento inicial de mudas de mentrasto "forma florífera". Ciência Agronômica, v.34, n.1, p.5-10, 2003.

NICOLOSO, F.T.; FORTUNATO, R.P.; FOGAÇA, M.A.F. Influência da posição da estaca no ramo sobre o enraizamento de Pfaffia glomerata (Spreng.) Pedersen em dois substratos. Ciência Rural, v.29, n.2, p.277-83, 1999.

OLIVEIRA, G.L. et al. Enraizamento de estacas de Lippia sidoides Cham. utilizando diferentes tipos de estacas, substratos e concentrações do ácido indolbutírico. Revista Brasileira de Plantas Medicinais, v.10, n.4, p.12- 7, 2008. PASQUAL, M. Cultura de tecidos. Lavras: UFLA/FAEPE, 2001. 165p.

PIMENTA, M.R. et al. Floração, germinação e estaquia em espécies de Lippia L. (Verbenaceae). Revista Brasileira de Botânica, v.30, n.2, p.211-20, 2007.

RAMOS, J.D. et al. Enraizamento de estacas herbáceas de 'mirabolano' (Prunus cerasifera EHRN) em diferentes substratos e concentrações de ácido indolbutírico. Revista Brasileira de Fruticultura, v.25, n.1, p.189-91, 2003.

SALOMÃO, L.C.C. et al. Propagação por estaquia dos maracujazeiros doce (Passiflora alata Dryand.) e amarelo ( $P$. edulis f. flavicarpa $O$. Deg.). Revista Brasileira de Fruticultura, v.24, n.1, p.163-7, 2002.

SIGNOR, D. et al. Estaquia herbácea de orégano. Scientia Agraria, v.8, n.4, p.431-4, 2007.

TAIZ, L.; ZEIGER, E. Fisiologia vegetal. 3.ed. Porto Alegre: Artmed, 2004. 719p. 